\title{
A method for separating the granulosa cells, the basal lamina and the theca of the preovulatory ovarian follicle of the domestic fowl (Gallus domesticus)
}

\author{
A. B. Gilbert, A. J. Evans, M. M. Perry and M. H. Davidson \\ A.R.C. Poultry Research Centre, King's Buildings, West Mains Road, \\ Edinburgh EH9 3JS, U.K.
}

The preovulatory follicle of the domestic hen is almost certainly a steroid-producing structure (see Shahabi, Norton \& Nalbandov, 1975). However, the details of steroidogenesis are unknown because studies are complicated by the presence of steroidogenic cells of two types, the granulosa and the thecal interstitial cells (Text-fig. 1). Furthermore, it is difficult to obtain preparations free from large amounts of yolk which interfere with the analytical techniques.

A simple method has been developed for separating granulosa cells from the surrounding thecal covering and to obtain them essentially free of yolk. This method also provides a procedure for isolating the basal lamina.

The hens used were from various commercially-available strains and were housed in cages or pens: the husbandry practice appeared not to affect the results obtained.

Preovulatory follicles were carefully excised from the ovaries of birds under general anaesthesia or killed by an overdose of sodium pentobarbitone (Nembutal: Abbott Laboratories). Best results were obtained with follicles weighing between 8 and $15 \mathrm{~g}$. The stalk of the excised follicle was held with forceps so that the clear, avascular stigma was uppermost (Gilbert, 1971). A cut, about $2 \mathrm{~cm}$ long, was made with a scalpel approximately along the line of the stigma, though the exact position is not critical. This step must be carried out quickly, with one sweeping stroke, and it should be completed before much escape of yolk occurs. Scissors may be used instead of a scalpel but they were found to be less satisfactory because there was a tendency for a point to penetrate too deeply into the yolk mass. Immediately after it had been cut the follicle was inverted over a suitable dish containing an aqueous medium and the follicular contents (yolk, perivitelline layer, granulosa and basal lamina) (Gilbert, 1971) were allowed to fall into the medium. The choice of medium depended on the purpose for which the granulosa cells were being harvested.

If the operation has been done correctly, the entire theca (Text-fig. 1, Pl. 1, Fig. 1b) remains held in the forceps, without contamination by yolk or granulosa material, and it can be used for studies of thecal activity. The yolk, covered by the perivitelline layer, the granulosa layer and the basal lamina (Text-fig. 1, Pl. 1, Fig. 3), settles as an almost undisturbed sphere on the floor of the vessel. The split occurs at a position different from that occurring during ovulation when the granulosa cells and the basal lamina remain with the theca (Pl. 1, Fig. 1a).

Removal of the granulosa layer, together with its associated basal lamina and perivitelline layer, from the yolk was carried out under a low-power lens or dissecting microscope with a black background. The cut ends of the composite membrane surrounding the yolk were located, grasped with fine dissecting forceps and gently pulled away from the yolk thereby everting the membrane over the surface of the yolk: this was made easier by using the yolk as a mass to pull against. Care was taken to protect the yolk from undue disturbance because yolk material dispersed throughout the medium prevented clear observation and caused unnecessary contamination of the preparation. It proved possible to remove the composite membrane in one piece with a very small amount of adherent yolk: when it was necessary to remove larger masses of yolk, the membrane was washed with a stream of medium from a Pasteur pipette.

The method provides large sheets of living granulosa cells sandwiched between the basal lamina and the perivitelline layer (PI. 1, Figs. 2 and 3); staining with Trypan Blue and Nigrosin revealed very few $(<5 \%)$ dead cells. 


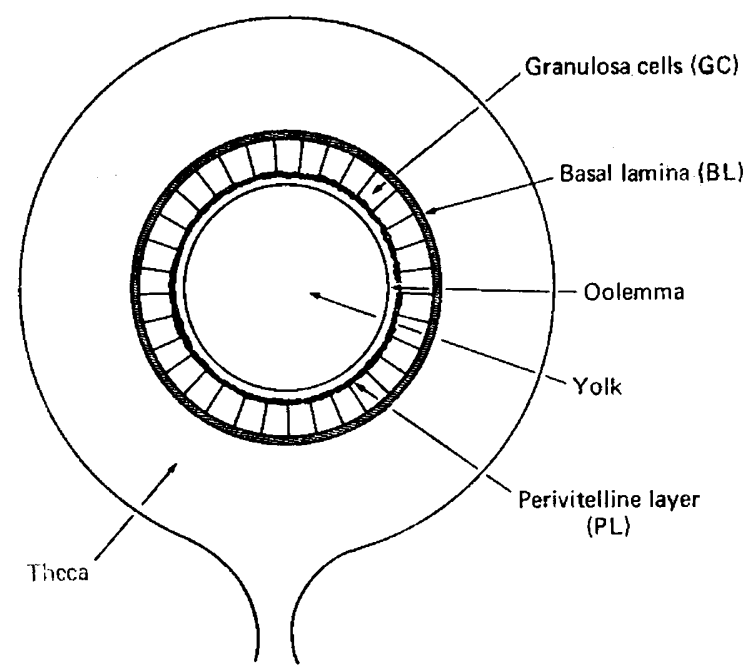

Text-fig. 1. Simplified diagram of a section through the mid-line of a preovulatory ovarian follicle of the domestic hen. The relative proportions of the layers are not to scale. After the incision has been made the contents of the follicle listed on the right-hand side are allowed to fall under gravity into the dish containing the aqueous medium (see text).

The preparation was checked for cleanliness by examining conventionally fixed and sectioned material in the electron microscope. On its outer aspect the basal lamina was completely free of thecal cells, whereas the perivitelline layer was contaminated to a variable extent by oocyte debris consisting of vesicular material, yolk granules and remnants of the oolemma (Pl. 1, Fig. 3). The granulosa cells, arranged in a single layer but separated from each other by gaps, were covered with numerous microvilli which, at the cell apices, extended into the meshwork of dense, rod-shaped elements of the perivitelline layer. The basal surfaces of the cells were comparatively smooth and were closely associated with the basal lamina. Details of the structure will be described in a later publication.

Isolated granulosa cells were obtained by separating the basal lamina from the perivitelline layer. This is best done under a dissecting microscope with magnifications of about $\times 50-100$ : very fine watch-maker's forceps were used to tease the basal lamina from the granulosa cells, which tended to remain attached to the perivitelline layer. Once separation had started, it was possible to pull the basal lamina away in large sheets, exposing the granulosa cells. These were then washed from the perivitelline layer by gentle agitation or a stream of medium from a Pasteur pipette. The granulosa cells were then collected by gentle centrifugation.

This technique is being used in several pieces of research, including an investigation of the physical and biochemical properties of the basal lamina and an examination of the detailed structure of the granulosa cells with the electron microscope. Tissue-culture work is being developed for study of yolk-transport mechanisms and steroidogenesis in granulosa cells and thecal interstitial cells.

\section{EXPLANATION OF PLATE 1}

Fig. 1. Conventional histological section of the hen theca stained with Mallory. (a) A normal discharged postovulatory follicle with intact granulosa (GC). (b) Preparation after removal of the granulosa, basal lamina and perivitelline layer, as described in the text. Scale bar $=100 \mu \mathrm{m}$.

Fig. 2. Preparation of hen granulosa cells with the basal lamina and perivitelline membrane. Most of the cells are apparently alive, though a few dead cells (DC) are visible. Trypan blue, $\times 500$.

Fig. 3. Electron micrograph of a section at right angles to the composite membrane fixed after stripping from the oocyte. A granulosa cell is seen sandwiched between the basal lamina (BL) and the perivitelline layer (PL). Sçale bar $=5 \mu \mathrm{m}$. 
PLATE 1
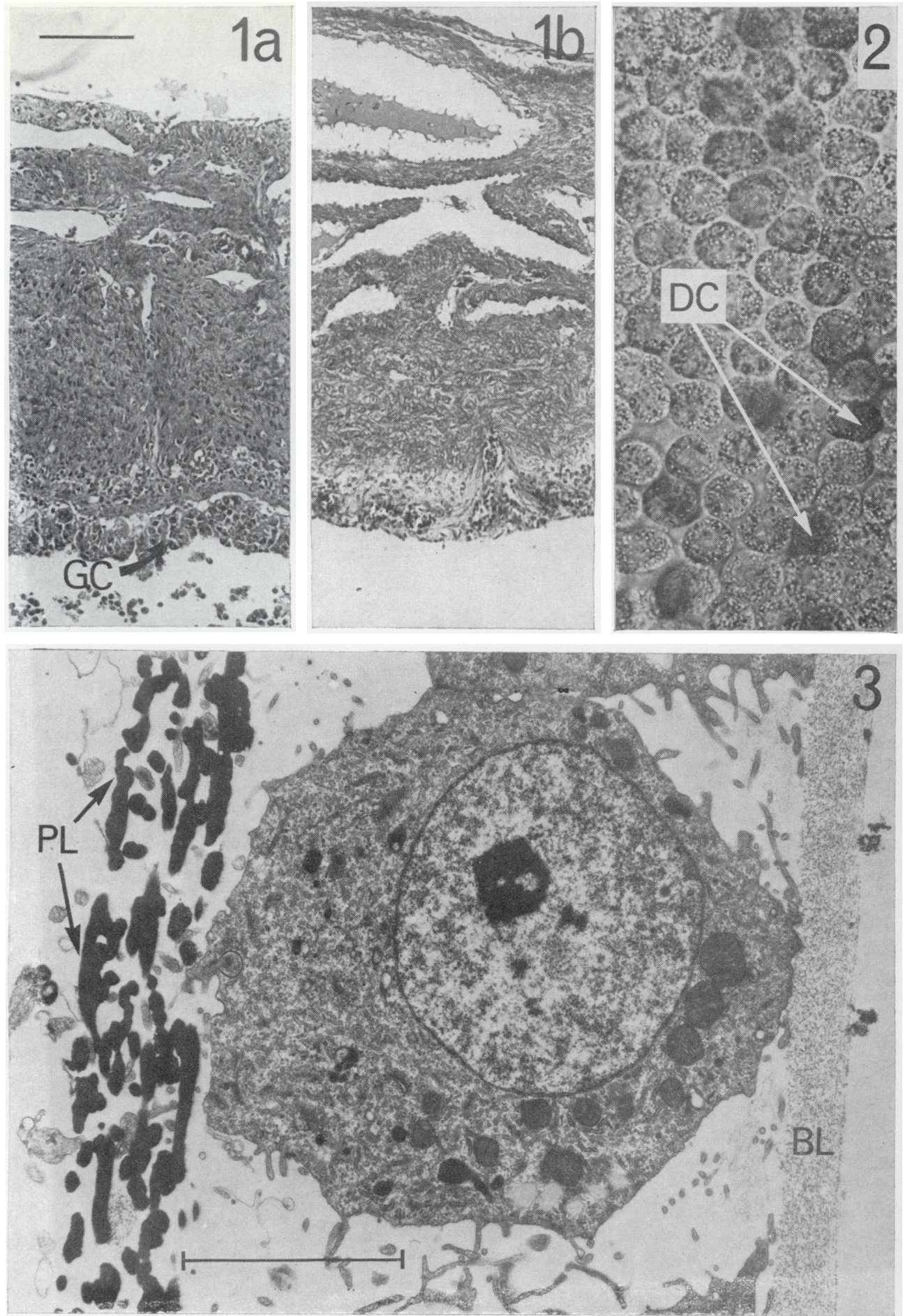


\section{References}

Gilbert, A.B. (1971) The ovary. In Physiology and Biochemistry of the Domestic Fowl, Vol. III, pp. 11631208. Eds D. J. Bell \& B. M. Freeman. Academic Press, London.
Shahabi, N.A., Norton, H.W. \& Nalbandov, A.V. (1975) Steroid levels in follicles and the plasma of hens during the ovulatory cycle. Endocrinology 96, 962-968.

Received 13 December 1976 